\title{
Design of the Tunnel Robot Based on the Crawler Chassis
}

\author{
Wei Song ${ }^{1,}$,, Xiaolong Zhou ${ }^{1}$, Ying Zhang ${ }^{1}$, Jie Xiao ${ }^{2}$ \\ ${ }^{1}$ State Grid Chongqing Electric Power Co. Electric Power Research Institute, 401123, Chongqing, \\ China
}

${ }^{2}$ The Smartech Institute, 518057, Shenzhen, China

a270642662@qq.com

Keywords: Crawler Chassis, Robot, Adaptation, Modular Joint, Swing Arm.

\begin{abstract}
In order to promote the movement inside tunnel robot in the cable tunnel and obstacle capability, designed a swing arm type crawler chassis, applied to the robot. The chassis adopts the modular design, assembly simple replacement maintenance convenience, enhances the terrain adaptability. Swing arm type caterpillar chassis adopts the former independent form of swing arm, using crawler swing arm Angle before climbing movement, before the swing arm can rotate at any Angle, boosting the terrain adaptability. Two walking tracks the independent motor drive, can achieve differential steering, a is a counter can be realized in situ $360^{\circ}$ rotation, can make the chassis in the narrow space free to; And simplify the traditional crawler chassis production complexity, strong load capacity at the same time.
\end{abstract}

\section{Introduction}

In order to achieve high quality and high reliability of power supply, the long distance transmission and distribution of electricity in the city mainly uses the high voltage cables in the tunnel, the safe and stable operation directly affects the city's production and life. Cable placed in the tunnel, due to the impact by wet environment, material aging, corrosive gases and other natural environment, cable broken skin, wear, corrosion or other damage, if not repaired in time, may bring off a large area and huge economic losses. Therefore, power companies need to regularly for transmission lines inspection, early detection of cable damage and defects, the first time make line maintenance and replacement, eliminate hidden dangers, the occurrence of accident prevention, to ensure the normal operation of the safety of power supply and power system.

Tunnel robot cruise along the tunnel, the need to cross the anti continuous steps, barriers, water, silt, and a variety of obstacles within the tunnel. In order to realize the inspection tasks of the robot, this paper introduces a design of a tunnel robot based on the track chassis. At present, the crawler chassis is the key research direction of the robot research institute at home and abroad. Mine, explosion-proof, and the detection of the robot is basically a robot based on the chassis of the robot. The front swinging arm type track chassis is convenient to change, simple structure and high terrain adaptability, and can be turned in a small space to make the robot move more flexible [1].

\section{Mechanism design}

\subsection{Master Plan Design.}

The tunnel robot is divided into mobile chassis and PTZ two parts as shown in Fig 1, mobile chassis for cruise, barrier, mobile and flexible, Yuntai responsible for inspection and monitoring circuit.

\subsection{Chassis Structure.}

The mechanism, the track chassis is a core component, with the following functions: walking, turning, climbing the stairs, climbing, over the threshold. As shown in Figure 2, the chassis mechanism is mainly composed of swing arm motor, track, walking motor, front swing arm. Left and right arm of the dual output worm gear deceleration motor drive, when the obstacle to realize simultaneous uplift, so as to realize the mechanical synchronization and simplify the control. 
Walking motor and swing arm motor with sealed water shield, ensure the wading depth of 200-300mm, involving the depth of mud 50-100mm.

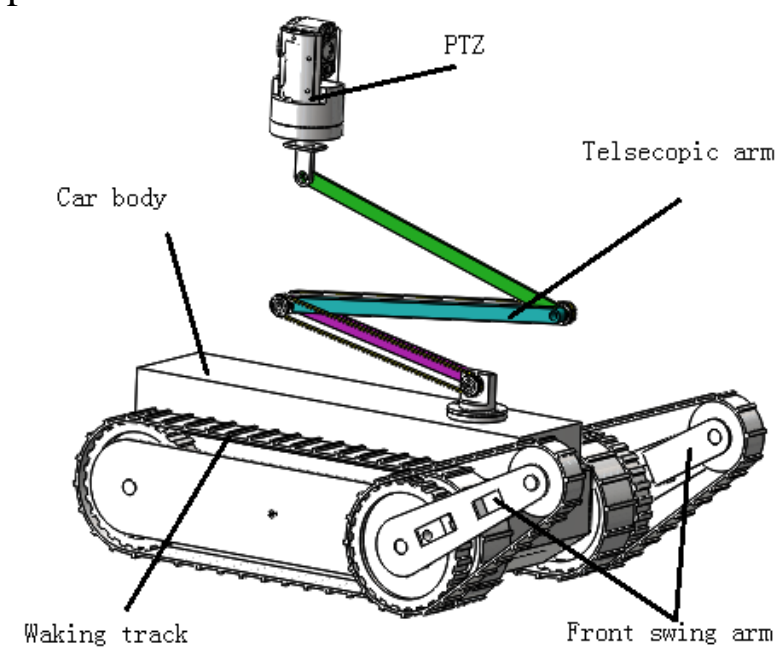

Fig.1 Whole mechanism

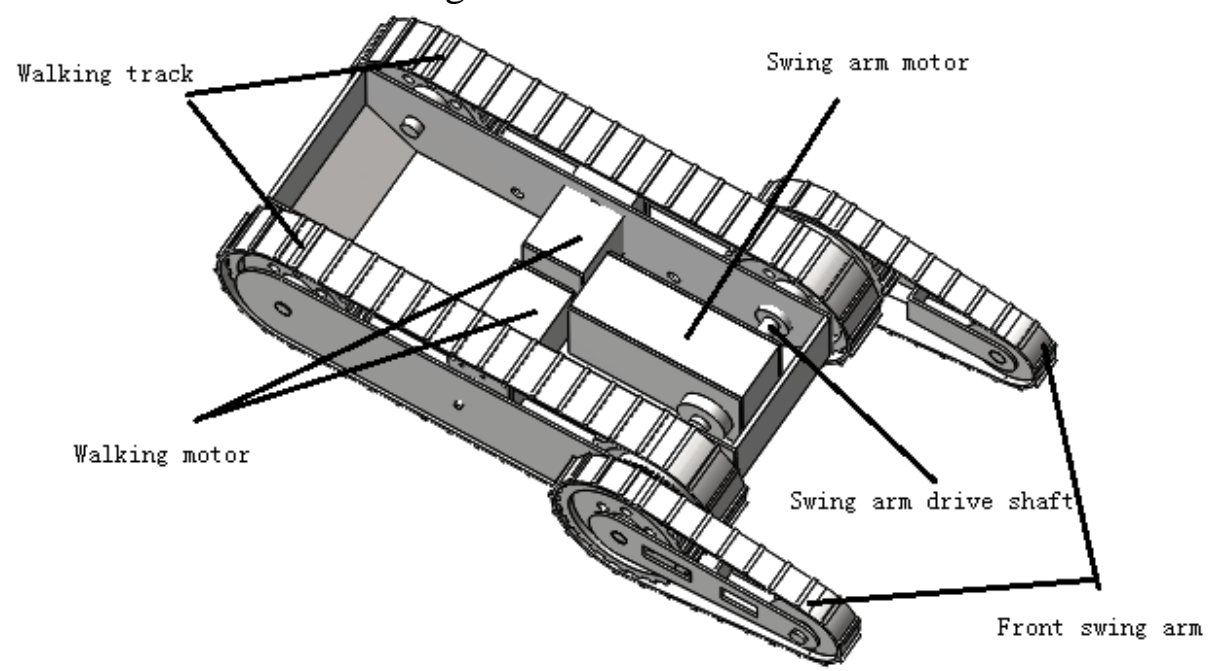

Fig.2 Undercarriage

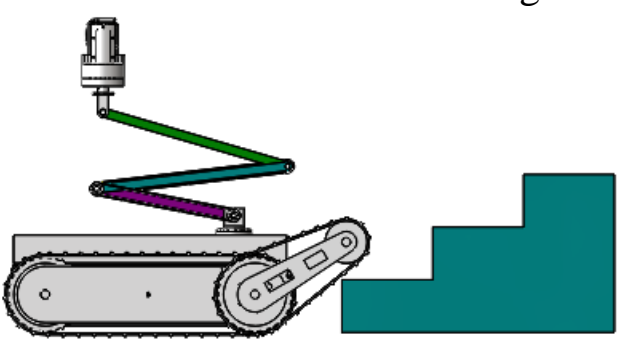

Fig.3 Obstacle

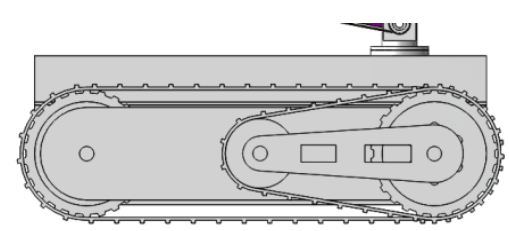

Fig.4 The swing arm retracted

Before walking arm retractable as shown in Figure4, theory turned on the spot diameter of 1000 $\mathrm{mm}$ as shown in Figure 5. Only in this way can guarantee in a very small space or channel can rotate, turn.

\subsection{Head structure.}

The servo lifting pan tilt system adopts three section folding arm, a motor drive, two synchronous belt transmission, to achieve three arm at the same time the folding and unfolding. This can reduce the number of motor and control difficulty.Advantages: simple structure, flexible lifting, a wide range of observation.Disadvantages: the structure occupies a little space, and more than a camera balance[2]. 


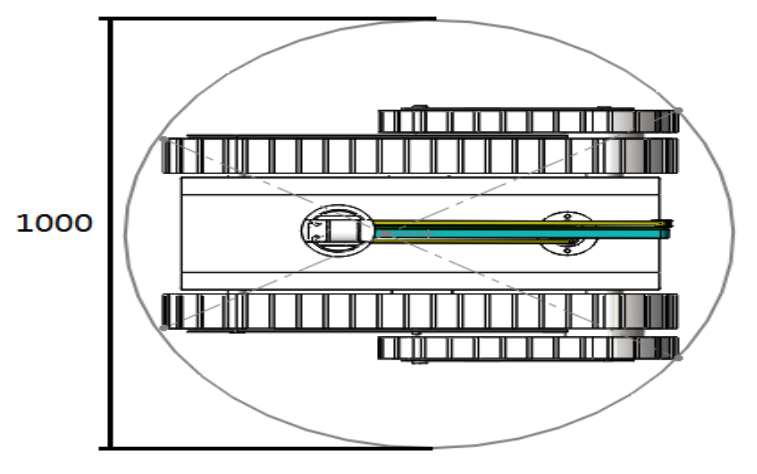

Fig.5 Turning diameter

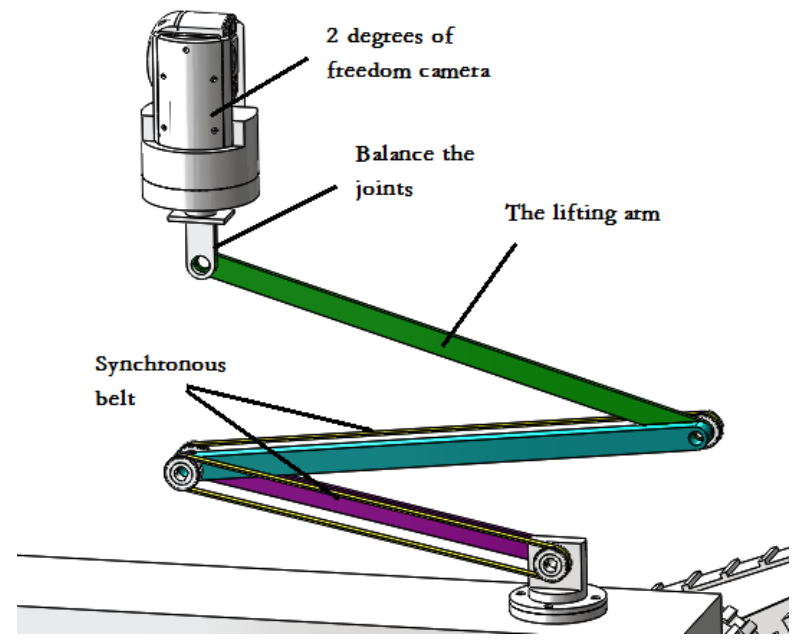

Fig.6 Head structure

\section{Kinematic analysis}

\subsection{Track Drive.}

The walking motor and the double driving wheel are driven by a chain, and the double driving wheel drives the walking track and the front swing arm track simultaneously, and the motor can drive the track to be inverted, as shown in Figure 7.

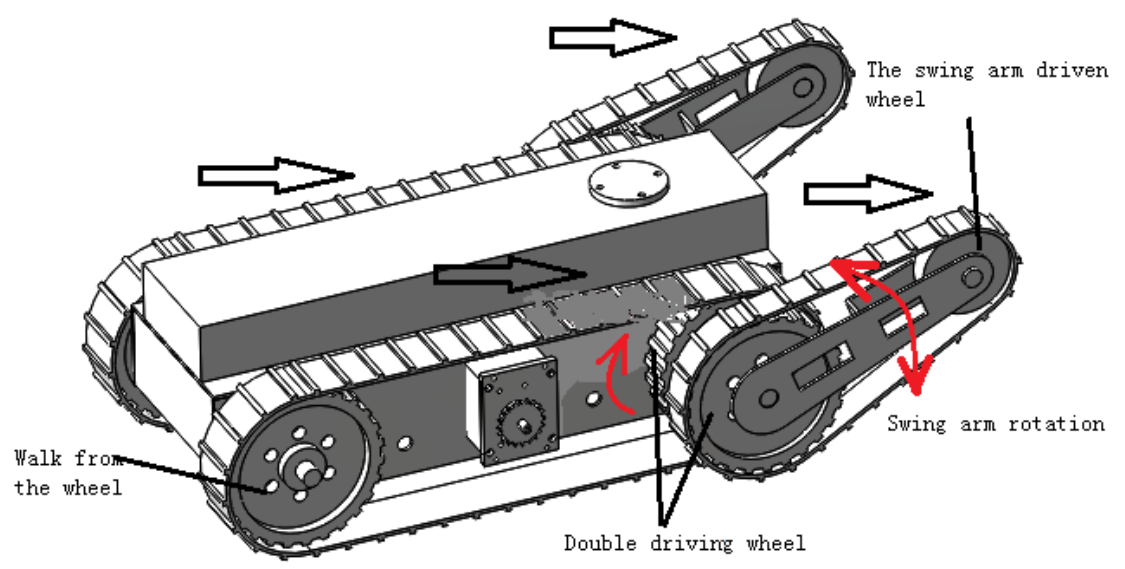

Fig.7 Track drive

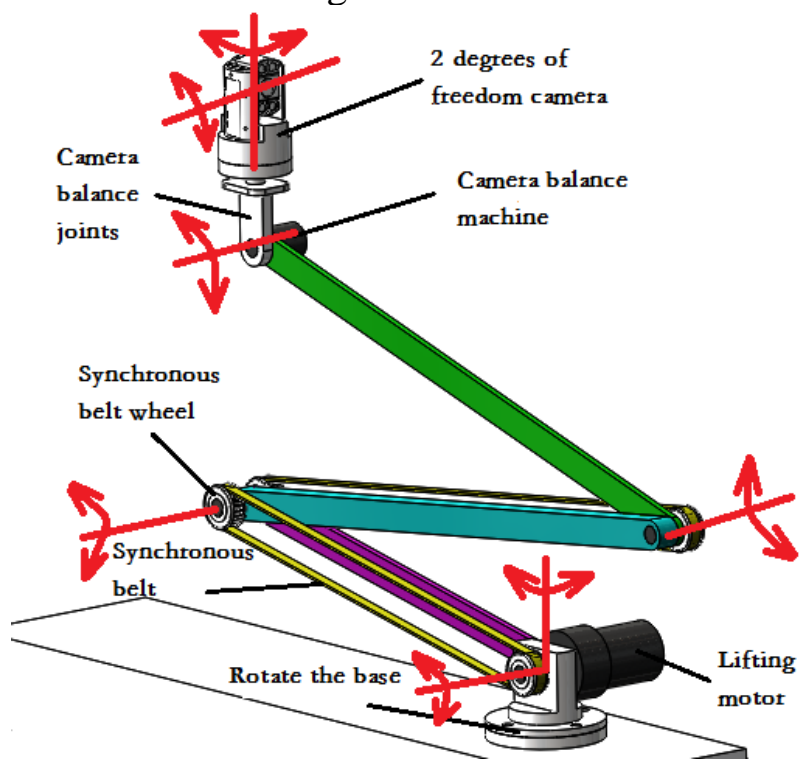

Fig.8 Pan degrees of freedom 


\subsection{Head Drive.}

7 degree of freedom platform, by five motor as shown in figure 8. Modular 2 degree of freedom camera to achieve the rotation and pitch, convenient viewing angle adjustment; camera balanced joint always maintain module of level of 2 degrees of freedom camera placed, to prevent shifting because of the lifting arm joint rotation caused dislocation of camera; lifting motor realize three section of the mechanical arm and lifting; rotating base with three sections of different lengths of mechanical arm can be realized camera, increase range of observations [3].

Summary of mechanical structure:

1 adaptable ability. Tunnel robot uses the swing arm crawler chassis, front swing arm can adjust angle, first of all on the continuous level tilt angle step highly good ability to adapt; and then swing arm can be folded and retracted, reduce the overall length of the crawler belt, can adapt the narrow space environment.

2 simplified structure and control. First is the simplification of the crawler chassis structure, relative and traditional crawler chassis, simplifying the guide and tensioning and damping structure; then 7 degree of freedom platform is applied with a lifting linkage, reducing the number of motor, and simplifies the control of the lifting algorithm, can achieve a key lifting.

In addition, shown in Figure 3-3 tilt structure, can see the lifting section three arm with different length, in order to give full play to the advantages of folding arm, with rotating base, can realize the camera moved, increase the range of camera observations.

\section{Conclusion}

Swing arm type crawler chassis is tunnel inspection robot obstacle crossing the main motion mechanism, the strong terrain adaptability and simplify the structure characteristics of robot is feasible to provide the safeguard, also the 7 degrees of freedom of a linkage lifting platform structure, making robot structure simpler, control is simple. In addition, the PTZ folding arm length difference, can realize the camera to a wide range of transfer, range of robot camera observations provides powerful guarantee. Based on the advantages of the tracked chassis, the tunnel inspection robot has a stronger adaptability to the task condition. Follow up will continue to optimize the overall structure of the tunnel robot, to further enhance the overall performance of the robot, to better provide services for the unmanned monitoring of the tunnel.

\section{References}

[1] Robots repair and examine live lines in serve condition [J]. Electrical World, 1989, 5:71-72.

[2] Pouliot, N., and Montambault, S. Field-Oriented Developments for LineScout Technology and its Deployment on Large Water Crossing Transmission Lines [J]. Journal of Field Robotics, 2012, 29(1): 25-46.

[3] Pouliot, N., and Montambault, S. Geometric Design of the LineScout, a Teleoperated Robot for Power Line Inspection and Maintenanc e[C]. IEEE International Conference on Robotics and Automation, Pasadena, CA, USA, 2008: 3970-3977. 